\title{
Directional Droplet Transport Mediated by Circular Groove Arrays. Part II : Theory of effect
}

Cong Liu ${ }^{1}$, Irina Legchenkova ${ }^{2}$, Libao Han ${ }^{1}$, Wenna Ge${ }^{1}$, Cunjing Lv ${ }^{3}$, Shile Feng ${ }^{1}$, Edward Bormashenko ${ }^{2 *}$, Yahua Liu ${ }^{1 *}$

${ }^{1}$ Key Laboratory for Precision and Non-traditional Machining Technology of Ministry of Education, Dalian University of Technology, Dalian 116024, China

${ }^{2}$ Chemical Engineering Department, Engineering Faculty, Ariel University, Ariel, 40700, Israel

${ }^{3}$ Department of Engineering Mechanics, Tsinghua University, Beijing 100084, China

As shown in Figure S1, we found that the spreading shape of droplet changed dramatically at $19 \leq W e<25$, which suggests that the wetting transition occur. Specifically, the value of maximum spreading angle $\theta_{\max }$ has a sudden jump at the transition range $19 \leq W e<25$. The theoretical transition point between Cassie-type bouncing and Wenzel-type bouncing are well verified by the experimental results (see Figure 2). As a result, we choose $W e=25$ as a switch in the following discussions.

a
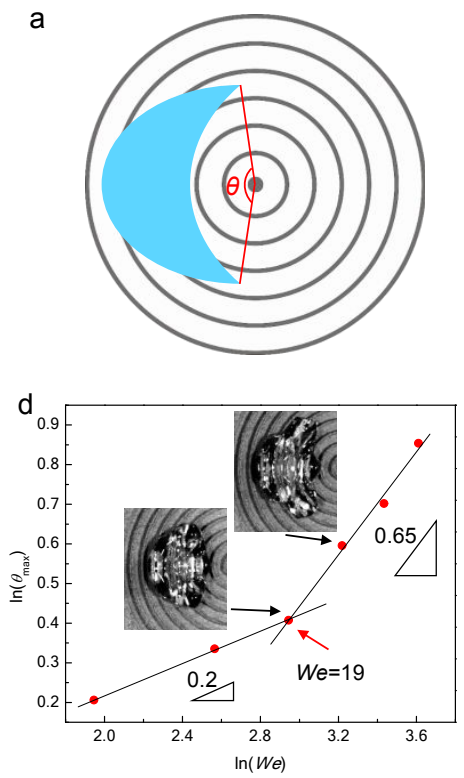
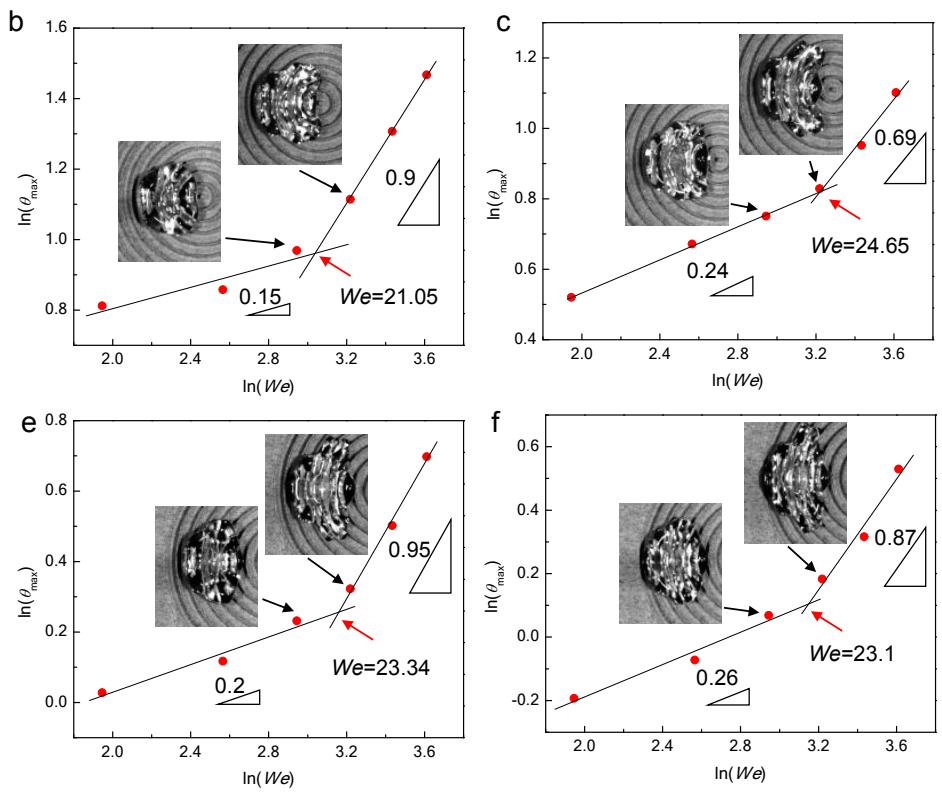

Figure S1. Geometrical parameters (a) and double logarithmic plots illustrating the time evolution of bouncing droplets shape on the W100S450H350 surface at $r=1.65 \mathrm{~mm}$ 
(b), $r=2.1 \mathrm{~mm}$ (c), $r=2.75 \mathrm{~mm}(\mathrm{~d}), r=3.3 \mathrm{~mm}$ (e), and $r=3.95 \mathrm{~mm}$ (f). Plot $\ln \theta_{\max }$ as a function of $\ln W e$ is plotted.

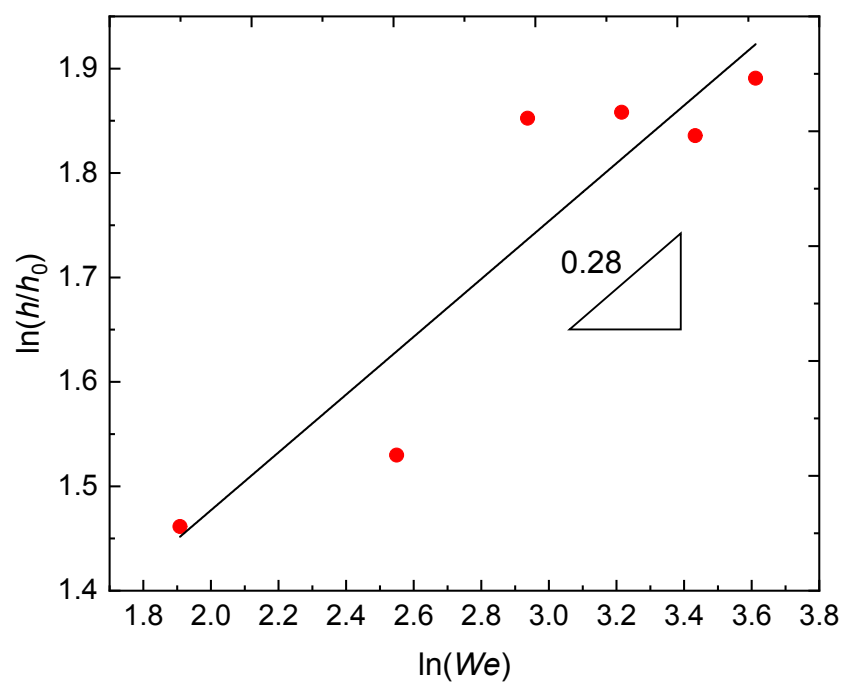

Figure S2. Double logarithmic plots illustrating the dependence of the rebouncing height $(h)$ on the Weber number $(W e)$ the W100S450H350 surface at $r=2.1 \mathrm{~mm}$. Plot $\ln \left(h / h_{0}\right)$ as a function of $\ln W e$ is plotted, $h_{0}=1 \mathrm{~mm}$. 